Document downloaded from:

http://hdl.handle.net/10251/82438

This paper must be cited as:

Esteban Romero, R.; Vincenzi, G. (2016). On generalised FC-groups in which normality is a transitive relation. Journal of the Australian Mathematical Society. 100(2):192-198. doi:10.1017/S1446788715000397.

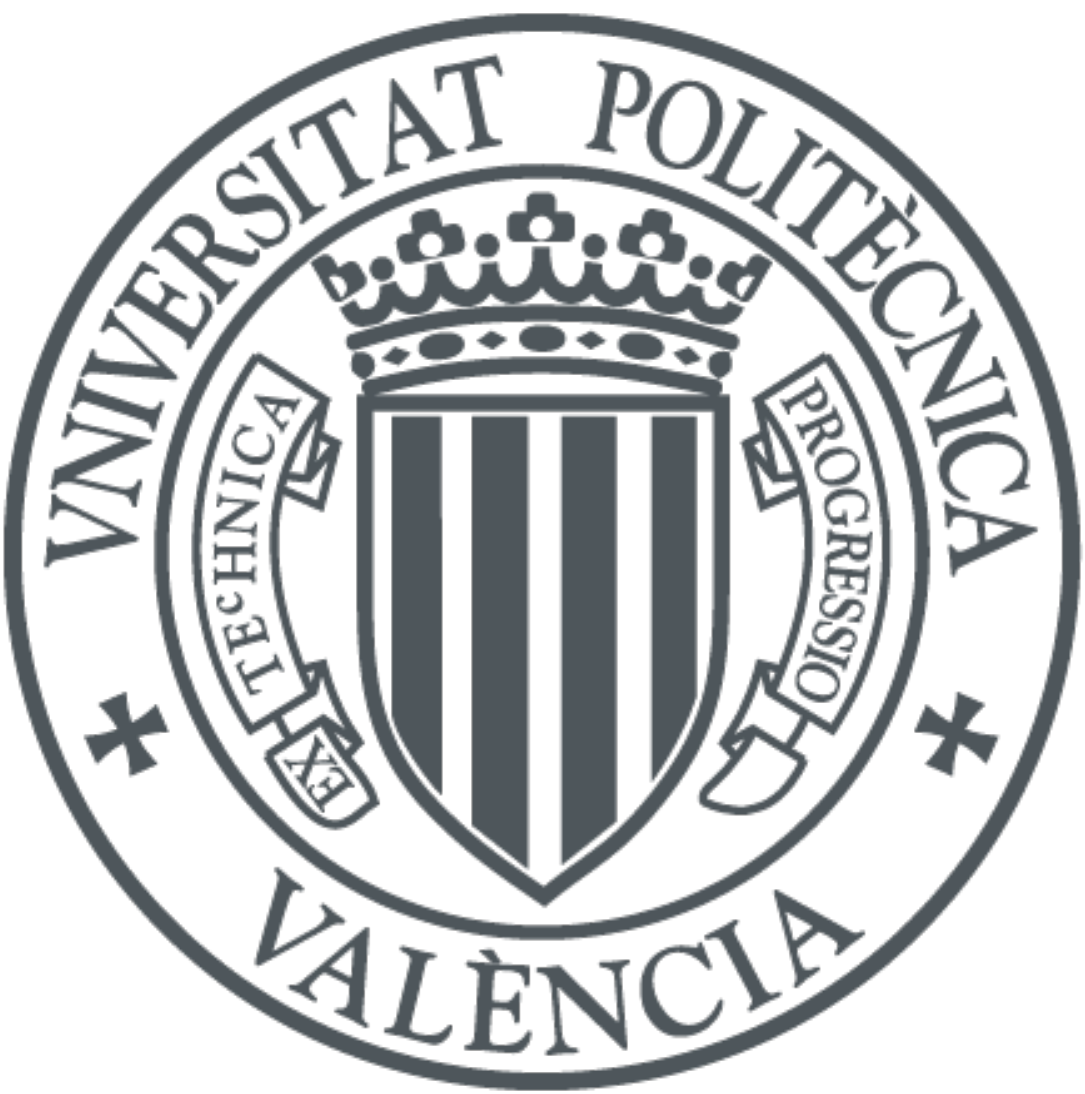

The final publication is available at

https://doi.org/10.1017/S1446788715000397

Copyright Cambridge University Press (CUP)

Additional Information 


\title{
ON GENERALISED FC-GROUPS IN WHICH NORMALITY IS A TRANSITIVE RELATION
}

\author{
R. ESTEBAN-ROMERO and G. VINCENZI
}

(October 9, 2015)

\begin{abstract}
We extend to soluble $\mathrm{FC}^{*}$-groups, the class of generalised FC-groups introduced in $[\mathrm{F}$. de Giovanni, A. Russo, G. Vincenzi, 'Groups with restricted conjugacy classes', Serdica Math. $J$. 28(3) (2002), 241-254], the characterisation of finite soluble T-groups obtained recently in [G. Kaplan, 'On T-groups, supersolvable groups and maximal subgroups', Arch. Math. 96 (2011), 19-25].

2010 Mathematics subject classification (Amer. Math. Soc.): 20F24, 20E34, 20F14, 20 F19. Keywords and phrases: T-group, FC-group, pronormal subgroup.
\end{abstract}

\section{Introduction}

A group in which normality is a transitive relation is called a T-group. In their seminal papers, G. Zacher [22] (1952) and W. Gaschütz [7] (1957) described the structure of finite soluble T-groups. Later, many authors studied finite groups in which normality is transitive, and more characterisations were obtained (see for instance $[1,2,3,9,10,14]$ ) in terms of properties of subgroups. It turns out that every finite soluble T-group is a $\bar{T}$-group (that is, a group in which every subgroup is a T-group). The description of infinite soluble groups in which normality is a transitive relation is more complex (see [15]), and examples of infinite soluble T-groups that are not $\overline{\mathrm{T}}$-groups can be constructed.

Following Kaplan [10], we say that $G$ has the $N N M$ property (nonnormal maximal) - in short: $G$ is an NNM-group - if each nonnormal subgroup of $G$ is contained in a nonnormal maximal subgroup of $G$. He obtained the following characterisation of finite soluble T-groups.

The first author has been supported by the research grant MTM2014-54707-C3-1-P from the Ministerio de Economía y Competitividad, Spain, and FEDER, European Union.

(c) XXXX Australian Mathematical Society 0263-6115/XX $\$$ A2.00 + 0.00 
Theorem 1.1 ([10, Theorem 1]). Let $G$ be a finite group. The following conditions are equivalent:

(1) $G$ is a soluble T-group.

(2) All subgroups of $G$ are NNM-groups.

In the same paper, Kaplan gives another characterisation of finite soluble T-groups. Following [10], for every subgroup $H$ of $G$, we denote by $\Lambda_{G}(H)$ the intersection of $G$ and all maximal subgroups of $G$ containing $H$, if there are any.

Theorem 1.2 ([10, Theorem 2]). Let $G$ be a finite soluble group. The following statements are equivalent:

(1) $G$ is a T-group.

(2) If $H$ is a nonnormal subgroup of $G$, then $\Lambda_{G}(H)$ is nonnormal in $G$.

The aim of this article is to extend the above results to some classes of generalised FC-groups. Recall that an FC-group is a group in which every element has finitely many conjugates.

Let $n$ be a nonnegative integer. The class $\mathrm{FC}^{n}$ is defined recursively as follows: $\mathrm{FC}^{0}$ is the class of all finite groups $G$, and a group $G$ belongs to the class $\mathrm{FC}^{n+1}$ if the factor group $G / \mathrm{C}_{G}\left(\langle x\rangle^{G}\right)$ belongs to $\mathrm{FC}^{n}$ for every element $x$ of $G$. We define the class $\mathrm{FC}^{*}$ of all $F C^{*}$-groups in the following way:

$$
\mathrm{FC}^{*}=\bigcup_{n \geq 0} \mathrm{FC}^{n}
$$

Clearly $\mathrm{FC}^{1}$-groups are just the $\mathrm{FC}$-groups, and for each nonnegative integer $n$, the class $\mathrm{FC}^{n}$ contains all finite groups and all nilpotent groups of class at most $n$. Moreover, we remark that the class of all $\mathrm{FC}^{*}$-groups is contained in the class of all FC-nilpotent groups [4, Theorem 3.2]. It can be seen that many of the properties common to finite and nilpotent groups also hold for $\mathrm{FC}^{*}$-groups. The theory of $\mathrm{FC}^{*}$-groups has been developed since their first appearance in $[4]$ (see $[8,18,19,21])$. Some characterisations of soluble $\mathrm{FC}^{*}$-groups in which normality is a transitive relation have been recently obtained (see [11, Theorem 5.2]); moreover, a weak form of transitivity of normality has been recently studied in the universe of all $\mathrm{FC}^{*}$-groups (see [20, Theorem 4.2]).

In this paper we will extend the quoted results obtained by Kaplan to the class of $\mathrm{FC}^{*}$-groups (see Theorems 2.4 and 2.5), obtaining new characterisations of soluble T-groups. Finally, an example is given to show that our characterisations do not hold either for FC-nilpotent or for hyperfinite groups.

Most of the notation is standard and can for instance be found in [16]. As a general reference for $\mathrm{FC}^{*}$-groups we cite [4]. 


\section{Statements and proofs of the results}

The following lemma describes two properties of subnormal subgroups of an $\mathrm{FC}^{*}$-group that will be useful in the sequel.

LEMma 2.1. Let $G$ be an $F C^{*}$-group, and $L$ be a subnormal subgroup of $G$.

(1) If $M$ is maximal in $G$ and $L \leq M$, then $L^{G} \leq M$.

(2) $\Lambda(L) \unlhd G$.

Proof. By hypothesis $G$ is an $\mathrm{FC}^{n}$-group for a non negative integer $n$.

(1). By [4, Theorem 3.2 and Corollary 3.3], the $n+1$-st term $\gamma_{n+1}(G)$ of the lower central series of $G$ is a torsion subgroup of the FC-centre of $G$ :

$$
\gamma_{n+1}(G) \leq \mathrm{FC}(G) .
$$

Thus we may consider a transfinite $G$-invariant chain

$$
N_{0}=\{1\} \triangleleft N_{1} \triangleleft \cdots \triangleleft N_{\delta}=\gamma_{n+1}(G)
$$

with finite factors such that $N_{\alpha+1} / N_{\alpha}$ is a minimal normal subgroup of $G / N_{\alpha}$ for $\alpha \leq \delta$. Suppose that $\gamma_{n+1}(G) \not \leq M$. Let $\mu=\min \{\alpha \leq \delta$ : $\left.N_{\alpha} \not \leq M\right\}$. Clearly $\mu$ is not a limit ordinal, and $\mu>0$. The choice of $\mu$ yields that $N_{\mu-1} \leq M$ and $N_{\mu} / N_{\mu-1}$ is a minimal normal subgroup of $G / N_{\mu-1}$. It follows from a result of Wielandt (see [16, Theorem 5.43]) that $N_{\mu} / N_{\mu-1}$ normalises all subnormal subgroups of $G / N_{\mu-1}$; on the other hand, $L N_{\mu-1} / N_{\mu}$ is subnormal in $G / N_{\mu-1}$ and $G=M \cdot N_{\mu}$ by the choice of $\mu$. Consequently:

$L^{G} N_{\mu-1} / N_{\mu-1} \leq\left(L N_{\mu-1} / N_{\mu-1}\right)^{G / N_{\mu-1}}=\left(L N_{\mu-1} / N_{\mu-1}\right)^{M / N_{\mu-1}} \leq M / N_{\mu-1}$.

Thus $L^{G} \leq M$. Hence we can assume that $\gamma_{n+1}(G) \leq M$. It follows that $M / \gamma_{n+1}(G)$ is a maximal subgroup of the nilpotent factor $G / \gamma_{n+1}(G)$, so that $M$ is normal in $G$, and again $L^{G} \leq M$. This completes the proof of (1).

In order to prove (2), we will slightly modify the arguments used in [10, Lemma 2.1(ii)]. In fact, if $L$ is not contained in any maximal subgroup of $G$, then $\Lambda_{G}(L)=G$, and the assertion is proved. On the other hand, if $M$ is a maximal subgroup of $G$ containing $L$, then it also contains $L^{G}$ by (i). It follows that

$$
\{M<\cdot G: L \leq M\}=\left\{M<\cdot G: L^{G} \leq M\right\},
$$

and $\Lambda_{G}(L)=\Lambda_{G}\left(L^{G}\right)$ is normal in $G$.

The next result extends the [10, Proposition 3] to the class of all $\mathrm{FC}^{*}$ groups. 
Proposition 2.2. Let $G$ be an $F C^{*}$-group such that for every nonnormal subgroup $H$ of $G, \Lambda_{G}(H)$ is not normal in $G$. Then:

(1) $G$ is a T-group.

(2) $G$ is an NNM-group.

Proof. By hypothesis $G$ is an $\mathrm{FC}^{n}$-group for a non negative integer $n$.

(1). Let $L$ be a subnormal subgroup of $G$, then $\Lambda_{G}(L)$ is normal in $G$ by Lemma 2.1. By hypothesis, $\Lambda_{G}(H)$ is not normal in $G$ for each nonnormal subgroup $H$ of $G$. Hence $L$ is normal in $G$. We have proved that $G$ is a T-group.

(2). Let $H$ be a nonnormal subgroup of $G$. By assumption $\Lambda_{G}(H)$ is not normal in $G$. In particular $\Lambda_{G}(H) \neq G$, and hence $H$ is contained in some maximal subgroup of $G$. Thus

$$
\Lambda_{G}(H)=\bigcap_{H \leq M<\cdot G} M,
$$

and there exists a maximal subgroup of $G$ containing $H$ which is not normal in $G$. We have proved that $G$ is an NNM-group.

In order to prove the extension of Theorems 1.1 and 1.2, we need a characterisation of $\mathrm{FC}^{*}$-groups in which normality is a transitive relation in terms of pronormal subgroups.

Recall that a subgroup $H$ of a group $G$ is said to be pronormal if for every element $x \in G$ the subgroups $H$ and $H^{x}$ are conjugate in $\left\langle H, H^{x}\right\rangle$, or, equivalently, there exists an element $y \in[H, x] \leq H[H, x] \leq\left\langle H, H^{x}\right\rangle$ such that $H^{y}=H^{x}$. It is well known that for finite groups, as well as for FC-groups, the property $\overline{\mathrm{T}}$ is equivalent to requiring all subgroups to be pronormal (see [14] and [5, Theorem 3.9]). This is not true in general: an example due to Kovács, Neumann and de Vries [12] shows that there exists a soluble periodic $\overline{\mathrm{T}}$-group $G$ whose Sylow 2 -subgroups are not pronormal. Moreover an example of Kuzennyi and Subbotin [13] shows that there are periodic metabelian groups whose primary subgroups are pronormal, and containing nonpronormal subgroups. Thus there are groups whose cyclic subgroups are pronormal, and containing nonpronormal subgroups. Nevertheless, for $\mathrm{FC}^{*}$-groups we have the following characterisation.

Theorem 2.3. Let $G$ be an $F C^{*}$-group. Then the following conditions are equivalent:

(1) $G$ is a soluble T-group.

(2) Every subgroup of $G$ is pronormal.

(3) Every cyclic subgroup of $G$ is pronormal.

When these conditions hold, $G$ is a metabelian $\bar{T}$-group and an FC-group. 
Proof. It is clear that (2) implies (3). Assume that $G$ satisfies (3), then $G$ is a $\overline{\mathrm{T}}$-group by [6, Lemma 3.2]. Thus $G$ is a T-group. It follows by [4, Theorem 4.6] (see also [19, Theorem 3.3]) that $G$ is an FC-group. Then each of the conditions (1), (2) and (3) implies that $G$ is an FC-group. By [5, Theorem 3.9], the conditions (1), (2) and (3) are equivalent.

Assume now that $G$ satisfies one of the conditions (1), (2) or (3) (and hence, all of them). By [15, Theorem 2.3.1], $G$ is metabelian.

We are now in position to prove an extension of Theorem 2 and Theorem 1 of [10] to the class of all $\mathrm{FC}^{*}$-groups.

Theorem 2.4. Let $G$ be a soluble $F C^{*}$-group. Then $G$ is a T-group if and only if for every nonnormal subgroup $H$ of $G, \Lambda_{G}(H)$ is not normal in $G$.

Proof. The condition is sufficient from Proposition 2.2.

Conversely, assume that $G$ is a soluble T-group. By Theorem 2.3, every subgroup of $G$ is pronormal, so that $G$ is a periodic group of the 'KuzennyiSubbotin'-type (see [17, Theorem E] or [13]). Thus:

(1) $G=B \ltimes A$, where $A$ is abelian and $B$ a Dedekind group.

(2) $\pi(A) \cap \pi(B)=\emptyset$ and $2 \notin \pi(A)$.

(3) Elements of $G$ induce power automorphisms in $A=[A, G]$.

(4) If $Q$ is a Sylow $\pi(G / A)$-subgroup of $G$, then $G=Q \ltimes A$.

Here, for a periodic group $X, \pi(X)$ denotes the set of the primes dividing the orders of the elements of $X$.

Let $H<G$, then $H=H_{1} \ltimes A_{1}$, where $A_{1}=A \cap H$ and $H_{1}$ is a Sylow $\pi(B)$-subgroup of $H$. By the condition (4), $H_{1}$ is contained in a complement $\bar{B}$ of $A$ in $G$, that is also a Dedekind group. Suppose that $H$ is not normal in $G$. Then $H / A_{1}$ is not normal in $G / A_{1}$, so that we can consider an element $h \in H_{1}$ such that $H / A_{1} \leq \mathrm{N}_{G / A_{1}}\left(\left\langle h A_{1}\right\rangle\right)<G / A_{1}$. By Theorem 2.3, $G / A_{1}$ is an FC-group, and it follows that the index $\left|G / A_{1}: \mathrm{C}_{G / A_{1}}\left(\left\langle h A_{1}\right\rangle\right)\right|$ is finite. Consequently $\mathrm{N}_{G / A}\left(\left\langle h A_{1}\right\rangle\right)$ has finite index in $G / A_{1}$, and hence there exists a maximal subgroup $M / A_{1}$ of $G / A_{1}$ containing $\mathrm{N}_{G / A_{1}}\left(\left\langle h A_{1}\right\rangle\right)$. On the other hand $\left\langle h A_{1}\right\rangle$ is subnormal in $\mathrm{N}_{G / A_{1}}\left(H / A_{1}\right)$, and by hypothesis $\mathrm{N}_{G / A_{1}}\left(H / A_{1}\right) \leq \mathrm{N}_{G / A_{1}}\left(\left\langle h A_{1}\right\rangle\right)$. Assume by contradiction that $\Lambda_{G / A_{1}}\left(H / A_{1}\right)$ is normal in $G / A_{1}$. Since $H$ is pronormal in $G$, the factor $H / A_{1}$ is pronormal in $G / A_{1}$, and an application of an elementary property of pronormal subgroups (see for example [6, Theorem 2.2]) shows that

$$
G / A_{1}=\mathrm{N}_{G / A_{1}}\left(H / A_{1}\right) \cdot \Lambda_{G / A_{1}}\left(H / A_{1}\right),
$$

which is contained in $M / A_{1}$. This contradiction shows that $\Lambda_{G / A_{1}}\left(H / A_{1}\right)$ is not normal in $G / A_{1}$, but clearly $\Lambda_{G / A_{1}}\left(H / A_{1}\right)=\Lambda_{G}(H) / A_{1}$, so that $\Lambda_{G}(H)$ is not normal in $G$. 
THEOREM 2.5. Let $G$ be an $F C^{*}$-group. The following statements are equivalent:

(1) $G$ is a soluble T-group.

(2) All the subgroups of $G$ are NNM-groups.

Proof. Assume that $G$ is a soluble T-group. By Theorem 2.3, every subgroup $H$ of $G$ is a soluble T-group. It follows by Theorem 2.4 that if $S$ is a nonnormal subgroup of $H$, then $\Lambda_{H}(S)$ is not normal in $H$. An application of Proposition 2.2 yields that $H$ is an NNM-group.

Assume now that all the subgroups of $G$ are NNM-groups. Note first that $G$ is an $\mathrm{FC}^{n}$-group for some non negative integer $n$, so that for every finitely generated subgroup $X$ of $G$, the factor $X / \mathrm{Z}_{n}(X)$ is finite and hence $X$ is polycyclic-by-finite, by [4, Proposition 3.6]. Consider now a finite homomorphic image $X^{\phi}$ of $X$. By hypothesis, every subgroup of $X^{\phi}$ is an NNM-group, and hence $X^{\phi}$ is a soluble T-group by [10, Theorem 1]. It follows that for every subgroup $L$ of $X$, the subgroup $L^{\phi}$ is pronormal in $X^{\phi}$ for every finite homomorphic image $X^{\phi}$ of $X$. An application of [5, Theorem 2.8] gives that $L$ is a pronormal subgroup of $X$. As $L$ is an arbitrarily chosen subgroup of $X$, we have that $X$ is a soluble T-group, by Theorem 2.3. On the other hand since the transitivity of normality is a local property (see [15, Corollary 2]), we conclude that $G$ is likewise a soluble T-group.

The following example shows that Theorems 2.4 and 2.5 do not hold either for FC-nilpotent groups or for hyperfinite groups.

EXAMPLE 2.6. Let $p$ be an odd prime, and let $G=\langle x\rangle \ltimes \mathbb{Z}_{p^{\infty}}$, where the element $x$ acts as the inversion over $\mathbb{Z}_{p^{\infty}}$. Clearly $G$ is a soluble T-group. Suppose now that $M$ is a maximal subgroup of $G$ containing the nonnormal subgroup $\langle x\rangle$. Then

$$
M=\langle x\rangle \mathbb{Z}_{p^{\infty}} \cap M=\langle x\rangle\left(\mathbb{Z}_{p^{\infty}} \cap M\right)<G,
$$

and thus the subgroup $\mathbb{Z}_{p^{\infty}} \cap M$ is one of the finite subgroups of $\mathbb{Z}_{p^{\infty}}$,

$$
1<H_{1}<\cdots<H_{n}<\cdots<\mathbb{Z}_{p^{\infty}},
$$

$H_{n}$ say. This yields a contradiction, as $M<\langle x\rangle H_{n+1}<G$.

We have showed that $\langle x\rangle$ is not contained in any maximal subgroup of $G$. Therefore $G$ is not an NNM-group. Moreover $\Lambda_{G}(\langle x\rangle)=G$, which is a normal subgroup of $G$, so that the set of all nonnormal subgroups of $G$ is not $\Lambda$-invariant.

Note also that $\mathbb{Z}_{p^{\infty}}$ is contained in the FC-centre of $G$, and so $G$ is FC-nilpotent. 
EXAMPLE 2.7. One might ask whether the class of all soluble T-groups coincides with the class of all $\mathrm{FC}^{*}$-groups $G$ such that $\Lambda_{G}(H)=H$ for each nonnormal subgroup $H$ of $G$. The answer is negative, even in finite groups. The dihedral group $G=\left\langle a, b \mid a^{9}=b^{2}=1, a^{b}=a^{-1}\right\rangle$ of order 18 has two conjugacy classes of nonnormal subgroups with representatives $D=\left\langle a^{3}, b\right\rangle$ and $C=\langle b\rangle$. We have that $D$ is a maximal subgroup of $G$ and $D$ is the unique maximal subgroup of $G$ containing $C$. In particular, $\Lambda_{G}(C)=\Lambda_{G}(D)=D$. Therefore $\Lambda_{G}(C) \neq C$. However, $G$ is clearly a soluble T-group.

\section{References}

[1] A. Ballester-Bolinches and R. Esteban-Romero, 'On finite $\mathcal{T}$-groups', J. Austral. Math. Soc. Ser. A 75 (2003), 1-11.

[2] A. Ballester-Bolinches, R. Esteban-Romero and M. Asaad, Products of finite groups, volume 53 of de Gruyter Expositions in Mathematics (Walter de Gruyter, Berlin, 2010).

[3] M. Bianchi, A. Gillio Berta Mauri, M. Herzog and L. Verardi, 'On finite solvable groups in which normality is a transitive relation', J. Group Theory (2) 3 (2000), $147-156$.

[4] F. de Giovanni, A. Russo and G. Vincenzi, 'Groups with restricted conjugacy classes', Serdica Math. J. (3) 28 (2002), 241-254.

[5] F. de Giovanni and G. Vincenzi, 'Pronormality in infinite groups', Math. Proc. R. Ir. Acad. (2) 100A (2000), 189-203.

[6] — 'Some topics in the theory of pronormal subgroups of groups', in: Topics in infinite groups, Quaderni di Matematica 8 (Aracne Ed. and Dept. Math. Seconda Univ. Napoli, Caserta, 2001) pp. 175-202.

[7] W. Gaschütz, 'Gruppen, in denen das Normalteilersein transitiv ist', J. Reine Angew. Math. 198 (1957), 87-92.

[8] D. Imperatore, A. Russo and G. Vincenzi, 'Groups whose proper subgroups are generalized FC-groups', J. Algebra Appl. (6) 10 (2011), 1301-1308.

[9] G. Kaplan, 'On finite T-groups and the Wielandt subgroup', J. Group Theory 14 (2011), 855-863.

[10] G. Kaplan, 'On T-groups, supersolvable groups, and maximal subgroups', Arch. Math. (Basel) (1) 96 (2011), 19-25.

[11] G. Kaplan and G. Vincenzi, 'On the Wielandt subgroup of generalized FC-groups', Int. J. Algebra Comput. (7) 24 (2014), 1031-1042.

[12] L. G. Kovács, B. H. Neumann and H. de Vries, 'Some Sylow subgroups', Proc. Roy. Soc. Ser. A 260 (1961), 304-316. 
[13] N. F. Kuzennyi and I. Ya. Subbotin, 'Groups in which all subgroups are pronormal', Ukr. Math. J. (3) 39 (1987), 251-254.

[14] T. A. Peng, 'Finite groups with pronormal subgroups', Proc. Amer. Math. Soc. 20 (1969), 232-234.

[15] D. J. S. Robinson, 'Groups in which normality is a transitive relation', Proc. Camb. Phil. Soc. 60 (1964), 21-38.

[16] - Finiteness conditions and generalized soluble groups (Springer, Berlin, 1972).

[17] D. J. S. Robinson, A. Russo and G. Vincenzi, 'On groups which contain no HNNextensions', Int. J. Algebra Comput. (7) 17 (2007), 1377-1387.

[18] , 'On the theory of generalized FC-groups', J. Algebra 326 (2011), 218-226.

[19] E. Romano and G. Vincenzi, 'Pronormality in generalized FC-groups', Bull. Austral. Math. Soc. 83 (2011), 220-230.

[20] , 'Groups in which normality is a weakly transitive relation', J. Algebra Appl. (1) 14 (2015), 1550007 (12 pages).

[21] A. Tortora and G. Vincenzi, 'The Engel elements in generalized FC-groups', Illinois J. Math. (to appear), arXiv:1412.6353.

[22] G. Zacher, 'Caratterizzazione dei t-gruppi finiti risolubili', Ricerche Mat. 1 (1952), $287-294$.

R. ESTEBAN-ROMERO, Institut Universitari de Matemàtica Pura i Aplicada, Universitat Politècnica de València, Camí de Vera, s/n, 46022 València (Spain) e-mail: resteban@mat.upv.es

Current address:

Departament d'Àlgebra, Universitat de València, Dr. Moliner, 50, 46100 Burjassot, València (Spain) e-mail: Ramon.Esteban@uv.es

G. VINCENZI,

Dipartimento di Matematica e Informatica, Università di Salerno, Via Giovanni Paolo II, Fisciano, 132, 84084 Salerno (Italy) e-mail: gvincenzi@unisa.it 